\title{
Cyclic Fluctuations in Coronary Blood Pressure and Flow Induced by Coronary Artery Constriction
}

\author{
Yasumi Uchida, M.D., Nobuo Yoshimoto, M.D., \\ and Satoru Murao, M.D.
}

\section{SUMMARY}

Slow cyclic fluctuations were frequently observed in blood pressure of the distal portion of the partially constricted coronary artery of anesthetized dogs. Lowering the mean peripheral coronary blood pressure below $50 \%$ of the control value was required for initiation of fluctuations. Each fluctuation in pressure was composed of 2 phases; the phase of rise and phase of fall. Almost invariably, the phase of rise and phase of fall were accompanied by increase and decrease in coronary blood flow, respectively. When the mean peripheral coronary blood pressure fell below $20 \%$ of the control value during the phase of fall, systolic bulge and elevation of the ST segment of surface ventricular electrocardiogram occurred. Period duration of each fluctuation ranged from $30 \mathrm{sec}$ to $14 \mathrm{~min}$. The cyclic fluctuations in pressure, flow, electrocardiogram and left ventricular wall motion were eliminated by nitroglycerin, but were not affected by phentolamine, propranolol, atropine, cervical vagotomy, and stellectomy.

\section{Additional Indexing Words:}

Coronary constriction Systolic bulge ST elevation Minute rhythm Cardiac denervation Nitroglycerin

HILE looking for experimental models for some type of clinical angina pectoris in dogs, we encountered a hitherto undescribed cyclic change $\mathrm{n}$ pressure and flow of the partially constricted coronary artery, which are frequently associated with ischemic changes phasically appearing in electrocardiogram and left ventricular motion. This paper describes this hitherto undescribed phenomenon.

\section{METHODS}

Experiments were carried out on 104 adult mongrel dogs under intravenous pentobarbital sodium anesthesia $(35-40 \mathrm{mg} / \mathrm{Kg})$. The trachea was intubated for artificial positive pressure respiration with air. The upper 7 ribs on the left side

From the Sccond Department of Internal Medicine, Faculty of Medicine, University of Tokyo, Tokyo, Japan.

Received for publication March 25, 1975. 
were removed and the anterior aspect of the heart was exposed by pericardiotomy. In many dogs, the proximal segment of the anterior descending branch of the left coronary artery was dissected free of surrounding tissues and a magnetic flowmeter was placed on it. Another segment about $5 \mathrm{~mm}$ distal to the magnetic flowmeter was also dissected and a screw clamp was placed on it for partial constriction. A force-displacement strain gauge arch was sewn to the anterior aspect of the left ventricle to monitor local left ventricular wall motion. A platinum-iridium electrode was fixed to the anterior wall of the left ventricle for recording a unipolar electrocardiogram. A small branch of the anterior descending branch which supplied the anterior apical area was dissected free and a catheter $1 \mathrm{~mm}$ in external diameter was inserted in retrograde fashion into it to measure the peripheral coronary blood pressure. In a small number of experiments, the proximal segment of the left circumflex branch and the right coronary artery were completely constricted by the threads for 1-2 min to examine changes in blood pressure and flow of the partially constricted anterior descending branch.

In 24 dogs (Experiment A), the anterior descending branch was completely constricted for $4 \mathrm{~min}$. Thereafter, the screw clamp was unfastened so as to set the peripheral blood pressure of the anterior descending branch (peripheral coronary blood pressure) above the level of complete constriction but below the level of control study. In 52 dogs (Experiment B), the anterior descending branch was partially constricted so as to set the peripheral coronary blood pressure below the level of before constriction but above the level of complete constriction. In 18 dogs, the anterior descending branch was completely constricted for 3-30 min and constriction was released by removing the screw clamp to examine whether the cyclic fluctuations in peripheral coronary blood pressure could occur.

In 5 dogs in which spontaneous cyclic fluctuations in peripheral coronary blood pressure and flow were produced, bilateral transection of the cervical vago-sympathetic trunks and the stellate ganglia was performed. Also, phentolamine (500 $\mu \mathrm{g}-1 \mathrm{mg} / \mathrm{Kg}$ ), propranolol $(500 \mu \mathrm{g}-1 \mathrm{mg} / \mathrm{Kg})$ and atropine $(50-100 \mu \mathrm{g} / \mathrm{Kg})$ were injected intravenously during the course of cyclic fluctuations in pressure and flow in 5,4 , and 5 dogs, respectively. In 4 dogs, a dose of $60 \mu \mathrm{g} / \mathrm{Kg}$ of nitroglycerin was injected intravenously to examine whether the agent could suppress the cyclic fluctuations.

\section{Results}

1. Thrce Experimental Methods for Producing Cyclic Fluctuations in Coronary Blood Pressure

A. Cyclic Fluctuations Induced by Partial Constriction following Complete Constriction (Experiment A)

The average mean systemic blood pressure was $142 \pm 13$ (mean $\pm S D$ ) $\mathrm{mmHg}$ in 29 dogs used in this series of experiments. The average mean peripheral coronary blood pressure was $108 \pm 9.5 \mathrm{mmHg}$ in control state. In 5 of these dogs, the blood flow through the anterior descending branch was measured by placing the flowmeter proximal to the screw clamp. In the 
other 16 dogs, the flowmeter was placed distal to the screw clamp. The systemic blood pressure, coronary blood pressure and flow in Experiment B were not different from those in Experiment A.

The peripheral coronary blood pressure was monitored for $30 \mathrm{~min}$ before constriction, however, cyclic fluctuations with a period duration in the minute range were not observed. After complete constriction for 3-5 min, the screw clamp was unfastened so as to set the peripheral coronary blood pressure below the control level but above the level of during complete constriction. An abrupt rise in peripheral coronary blood pressure and flow followed by a gradual decrease was observed immediately after unfastening the screw clamp (Fig. 1). After 3-15 (8.4 \pm 4.2$)$ min following unfastening, slow cyclic fluctuations in pressure and flow of the partially constricted coronary artery developed. In 15 experiments, in which peripheral coronary blood pressure was set below $50 \%$ of the control level, the fluctuations in pressure developed in 11, while they developed in only 4 of 14 experiments in which peripheral coronary blood pressure was set above $50 \%$. The fluctuations in coronary blood flow was recorded in 4 preparations in which fluctuations in peripheral coronary blood pressure developed. Precise analysis of cyclic changes in pressure, flow, direct lead electrocardiogram and left ventricular wall motion which were almost the same as those observed in Experiment $B$ were shown in one of the following sections.

B. Cyclic Fluctuations Induced by Partial Constriction (Experiment B) The anterior descending branch was partially constricted in 52 dogs. Following the procedure, the peripheral coronary blood pressure fell abruptly and thereafter rose gradually. The gradual rise in pressure was followed by a gradual fall, and the cyclic fluctuations developed in 14 of these preparations. The time from the beginning of constriction to development of the fluctuations was $4-31(15.0 \pm 10.4) \mathrm{min}$. The coronary blood flow began to fluctuate with pressure in these preparations. The fluctuations developed in 13 of 33 preparations in which peripheral coronary blood pressure was reduced below $25 \%$ of the control value by partial constriction. On the other hand, the fluctuations developed in only one of 19 preparations in which the peripheral coronary blood pressure was set above $25 \%$ by constriction. The difference in occurrence of the fluctuations between these 2 groups was significant $(\mathrm{P}>.05)$.

C. Cyclic Fluctuations Developed after Release of Complete Constriction (Experiment $\mathrm{C}$ )

The anterior descending branch was constricted completely for 3-30 min and was released by removing the screw clamp in 18 dogs. In these experiments, the coronary blood flow was not measured. The cyclic fluctuations 


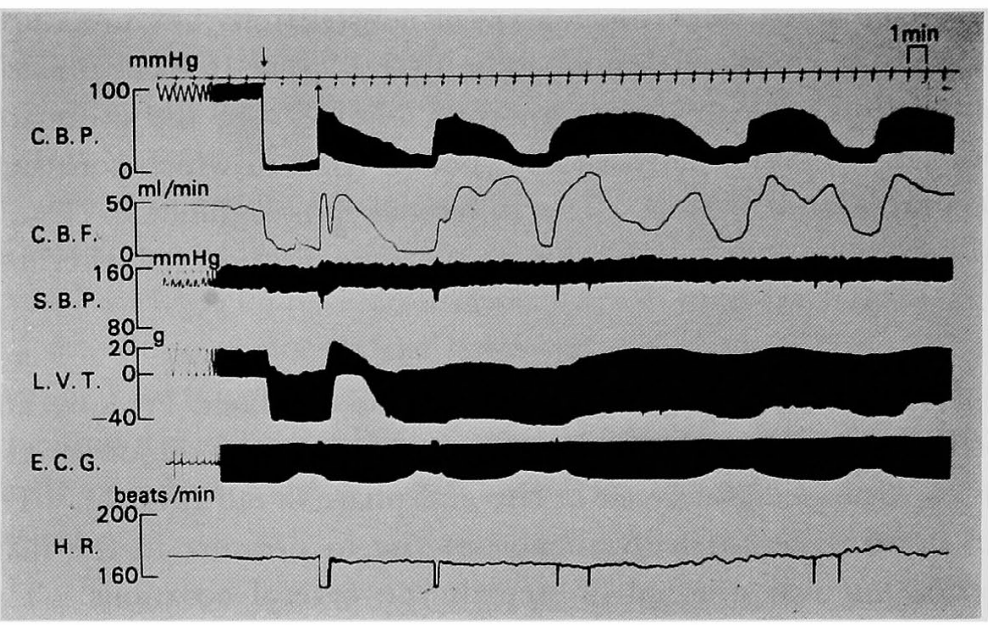

Fig. 1. Slow cyclic fluctuations in peripheral coronary blood pressure and flow induced by unfastening the screw clamp (upward arrow) following complete constriction (downward arrow) of the anterior descending branch. From the top channel: peripheral blood pressure of the anterior descending branch (C.B.P.), blood flow through the same branch (C.B.F.), systemic blood pressure (S.B.P), left ventricular tension (L.V.T.), surface electrocardiogram (E.C.G.), and heart rate (H.R.)

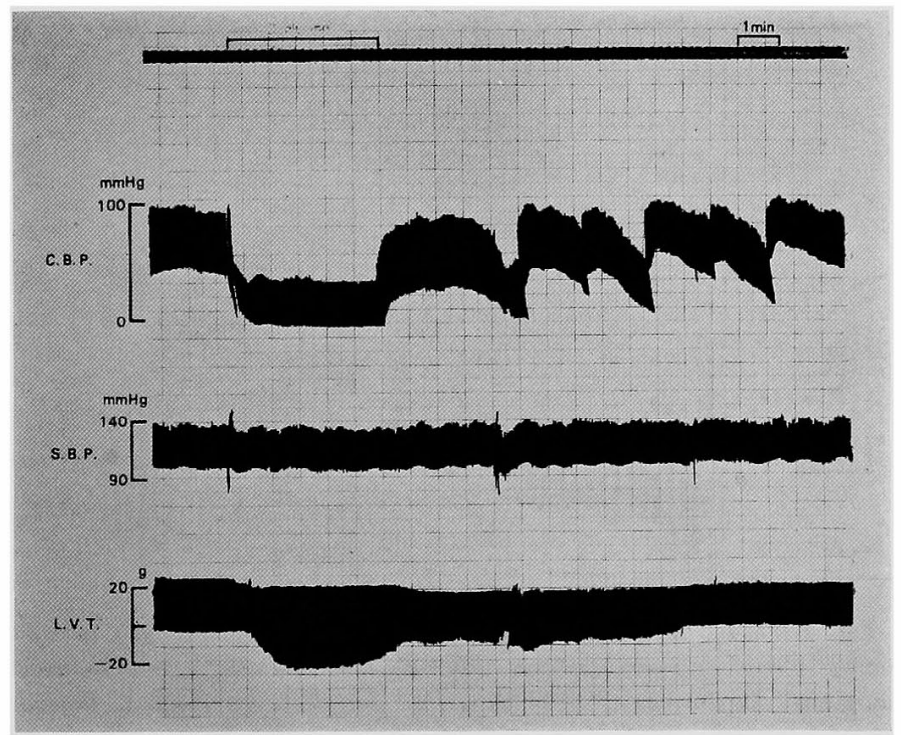

Fig. 2. Cyclic fluctuations in peripheral coronary blood pressure which developed after release of complete constriction (horizontal bar). 
in pressure developed after the release of constriction, but not during constriction. The fluctuations developed in $7(46.6 \%)$ of 15 trials in which complete constriction was performed for over $7 \mathrm{~min}$. On the other hand, the fluctuations developed in only $5(5.9 \%)$ of 82 trials in which constriction was performed for within $7 \mathrm{~min}$. The difference was significant $(\mathbf{P}<.01)$. In all these experiments, however, the fluctuations disappeared after 1-5 cycles (Fig. 2).

2. Mode of Cyclic Fluctuations in Coronary Blood Pressure and Flow Each cycle of fluctuations in peripheral coronary blood pressure was composed of 2 phases; the phase of rise and phase of fall (Fig. 1). The phase of rise in pressure began with an abrupt rise in pressure in the majority of experiments, but was gradual or stepwise on several occasions. The blood flow through the partially constricted coronary artery began to increase with the rise in pressure (Fig. 1). The flow frequently increased above the control level during the rise in pressure (Fig. 1). The phase of fall showed a gradual curve in pressure and flow in the majority of experiments, but began abruptly on several occasions (Fig. 7).

The mean peripheral coronary blood pressure of the 2 phases was measured in 24 preparations. The pressure was widely ranged in each phase;
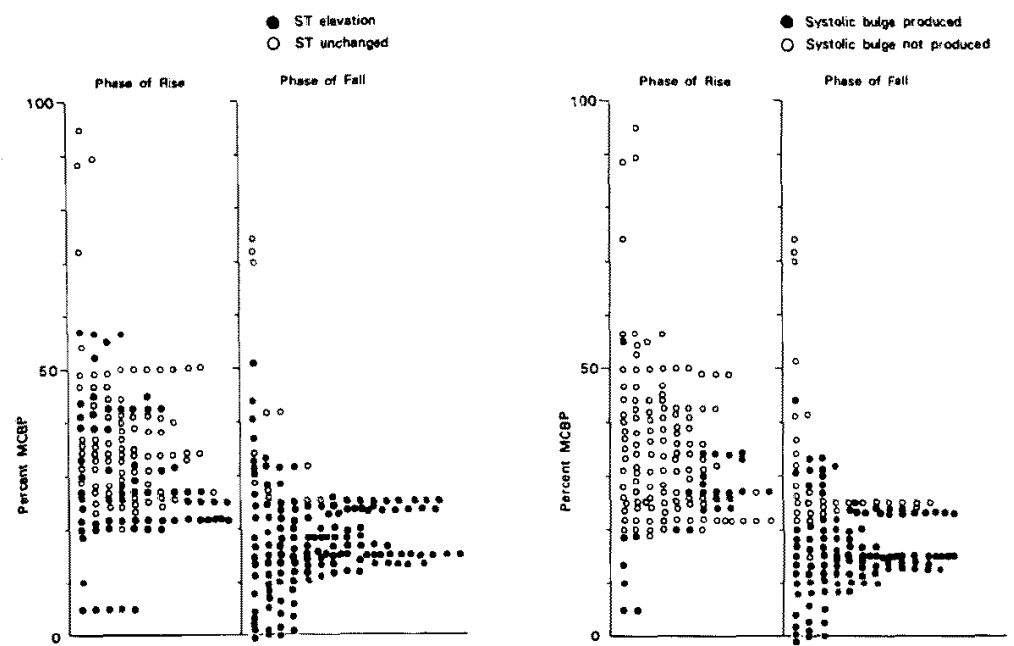

Fig. 3. Left half: Relationship between percent mean peripheral coronary blood pressure (percent MCBP) and changes in surface electrocardiogram. Open circles $=\mathrm{ST}$ elevation not occurred. Solid circles $=\mathrm{ST}$ elevation occurred. Right half : relationship between percent mean peripheral coronary blood pressure and changes in left ventricular wall motion. Open circles = systolic bulge not occurred. Solid circles=systolic bulge occurred. 
the peak mean pressure during the phase of rise was 5-95 and that during the phase of fall was $0-74 \%$ of the control value (Fig. 3). There was no obvious relationship between the fluctuations in coronary blood pressure and flow and the changes in systemic blood pressure and heart rate.

The period duration was measured in 145 fluctuations of 24 dogs. The duration was $30 \mathrm{sec}$ to $14 \mathrm{~min}$ and the frequency distribution was multi-peaked as shown in Fig. 4. The period duration was not strictly constant with variations of a few minutes during the course of observation. Although we did not determine how long the cyclic fluctuations could continue, the fluctuations were quite stable and persisted for hours in experiment $A$ and $B$ even when the systemic blood pressure and the heart rate changed during the couse of experiments.

3. Gyclic Ghanges in Surface Electrocardiogram and Local Left Ventricular Wall Motion Associated with Cyclic Fluctuations in Coronary Blood Pressure and Flow

The changes in electrocardiogram and left ventricular wall motion which were associated with cyclic fluctuations in coronary blood pressure and flow were shown in Fig. 5. The changes of these parameters were identical irre-

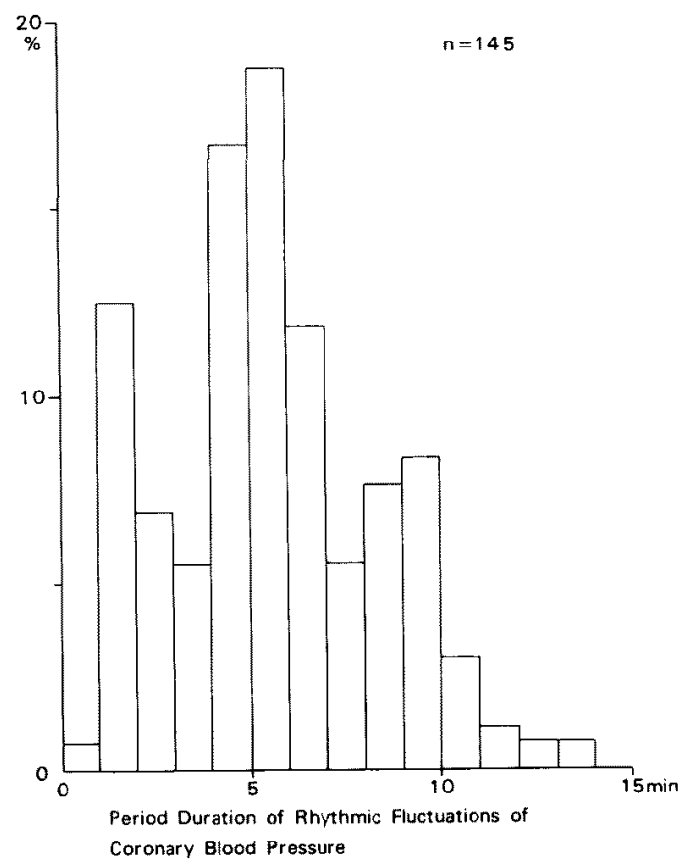

Fig. 4. Frequency distribution (in percent) of the period duration of cyclic fluctuations in peripheral coronary blood pressure. 


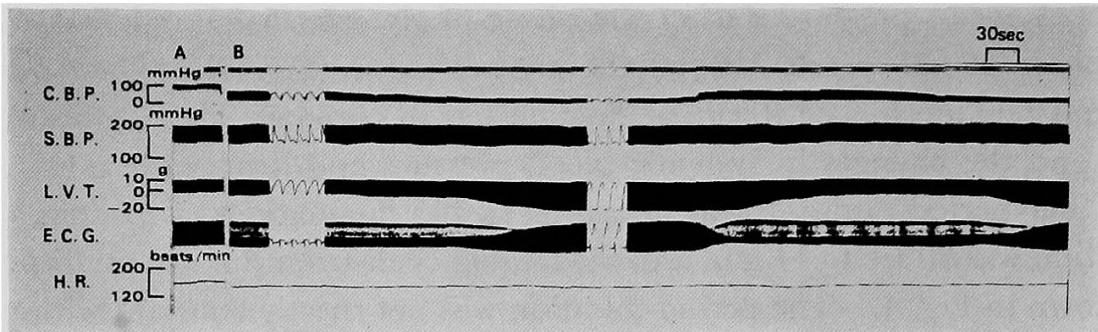

Fig. 5. Changes in left ventricular motion and electrocardiogram which are associated with cyclic fluctuation in peripheral coronary blood pressure. A. : control. B.: during constriction.

spective of the methods employed to produce cyclic changes. During the phase of fall, the level of the ST segment of electrocardiogram was elevated in 133 of 145 cycles. In the remaining cycles, the level of the segment was the same as that before coronary constriction. During the phase of rise, the level of the ST segment became the same as that before constriction in 53 cycles and elevation was reduced in its magnitude in 80 cycles. It was revealed that elevation of the segment occurred when the mean peripheral coronary blood pressure fell below $30 \mathrm{mmHg}$ ( $25 \%$ of the control value) during the phase of fall and that the segment remained elevated when the pressure did not rise over $30 \mathrm{mmHg}$ ( $30 \%$ of the control value) during the phase of rise (Fig. 3).

During the phase of fall, a decrease in left ventricular tension measured from the strain gauge arch during the phase of systole, the systolic bulge, developed in 85 cycles of fluctuations and it disappeared during the phase of rise. In 27 cycles, the bulge remained even during the phase of rise (Fig. 3). The cyclic changes in electrocardiogram and left ventricular wall motion always followed the cyclic fluctuations in pressure and flow.

4. Results of Interventions which Might Effect Initiation and Maintenance of the Cyclic Fluctuations

In 7 preparations, the effect of removal of the screw clamp was examined during the course of cyclic fluctuations. In all these preparations, the fluctuations with a smaller amplitude persisted at a higher level of the coronary blood pressure for 20 to $40 \mathrm{~min}$.

Simultaneous constriction of the left circumflex branch and the right coronary artery caused a fall in peripheral blood pressure of the partially constricted anterior descending branch as well as a fall in systemic blood pressure, however, the flow through the anterior descending branch was not reduced in all 5 preparations tested. Also, the cyclic fluctuations were not eliminated. 
Ventricular premature beats and pulsus alternans which frequently occurred during the course of cyclic fluctuations could not alter the rhythmicity. The changes in systemic blood pressure caused by artificial respiration and Traube-Herring-Meyer waves observed in several preparations seemed to have no relation to the cyclic fluctuations.

In order to examine whether the cardiac nerves played any role in initiation and maintenance of the cyclic fluctuations, the bilateral cervical vagotomy and stellectomy were performed. Although the systemic as well as the peripheral coronary blood pressure and flow were altered in their levels, the cyclic fluctuations persisted at a different level of pressure and flow.

The cyclic fluctuations in peripheral coronary blood pressure were also produced by partial constriction even after cervical vagotomy and stellectomy in 5 dogs.

Although the intravenous injections of phentolamine resulted in a fall in peripheral coronary blood pressure as well as in systemic blood pressure, the cyclic fluctuations remained. The intravenous injections of propranolol reduced the magnitude of the elevation of the ST segment and systolic bulge and caused a decrease in heart rate, while could not eliminate the cyclic fluctuations (Fig. 6). The intravenous injections of $60 \mu \mathrm{g} / \mathrm{Kg}$ of nitroglycerin during the course of cyclic fluctuations showed effects on the fluctuations depending on timing of the injections; the injections during the phase of rise

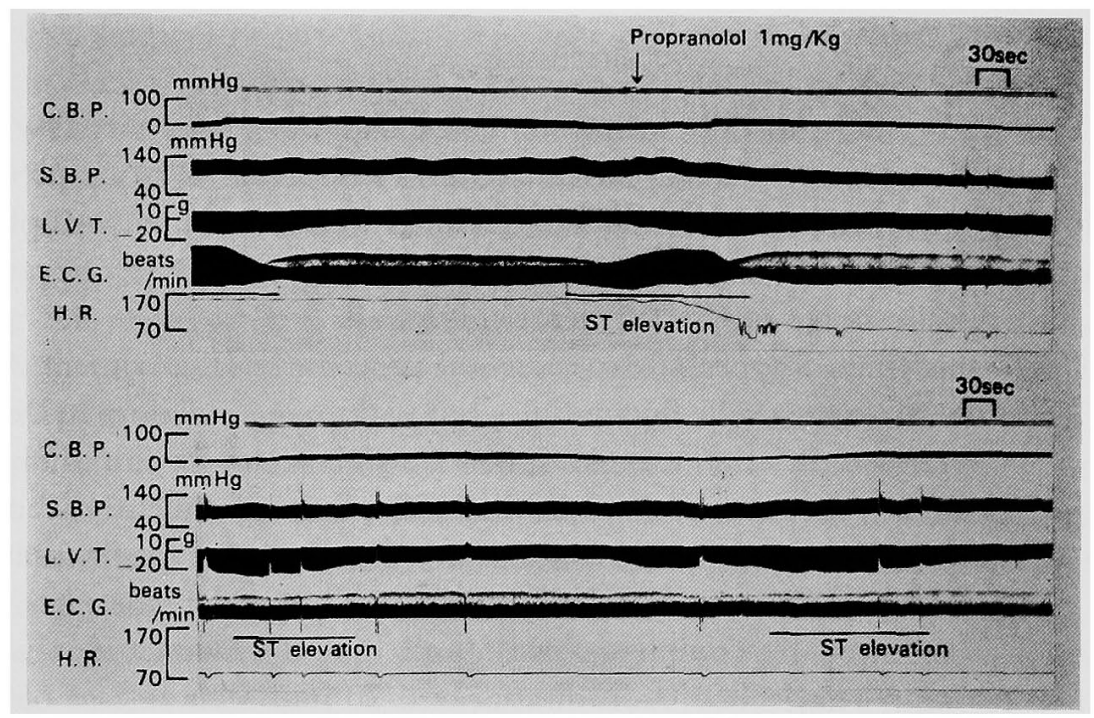

Fig. 6. The effect of intravenous injection of $1 \mathrm{mg} / \mathrm{Kg}$ propranolol on cyclic fluctuations in peripheral coronary blood pressure, left ventricular wall motion, electrocardiogram and heart rate. Upper half: before propranolol. Lower half: after propranolol. 


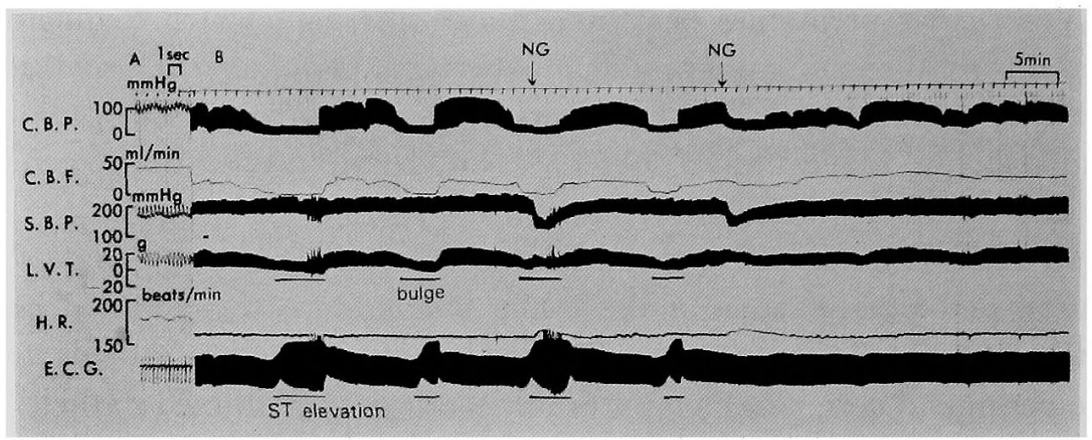

Fig. 7. The effect of intravenous injections of $60 \mu \mathrm{g} / \mathrm{Kg}$ of nitroglycerin (at downward arrows) on cyclic fluctuations. Systolic bulge and elevation of the ST segment of electrocardiogram were underlined. A: before constriction. B: during constriction.

reduced the amplitude of the fluctuations of both peripheral coronary blood pressure and flow by elevating the level of pressure and flow of the phase of fall, shortened the duration of each fluctuation, and suppressed or eliminated systolic bulge and elevation of the ST segment of electrocardiogram in all 4 dogs. The effect of the agent continued for up to $30 \mathrm{~min}$. On the contrary, the injections during the phase of fall could not eliminate nor suppress the fluctuations (Fig. 7). The minimum dose which could influence the fluctuations was not determined.

\section{Discussion}

This study indicates that, when the coronary artery was partially constricted, there could take place cyclic fluctuations in peripheral blood pressure and flow of the constricted coronary artery which were associated with phasic appearance or augmentation of elevation of the ST segment of electrocardiogram and systolic bulge of the left ventricular wall.

Up to this time, 3 types of experimental procedures (Experiment A, B, and $\mathrm{C}$ ) could initiate the cyclic fluctuations, but with frequent failure. Degree of coronary artery constriction seemed to be a possible factor for initiation and maintenance of the fluctuations, since the fluctuations more frequently occurred the more severe was the degree of constriction. The facts that the fluctuations could not persist over several cycles in Experiment $\mathrm{C}$ in which complete constriction was followed by complete release and that the fluctuations which persisted during partial constriction disappeared $30 \mathrm{~min}$ after release of constriction indicate that coronary constriction was an important factor for maintenance of the cyclic fluctuations.

Several mechanisms can be speculated as possible factors for the cyclic 
fluctuations in coronary blood pressure and flow; changes in systemic blood pressure, periodic changes in tone of smooth muscles of the coronary artery proximal to the point of pressure measurement, changes of tone of the more peripheral coronary arterial smooth muscles, periodic changes in collateral blood flow and cyclic changes in perivascular pressure. In this study, changes in systemic blood pressure and heart rate had no obvious relation to the cyclic fluctuations. On the other hand, rise and fall in peripheral coronary blood pressure were always accompanicd by an increase and decrease in coronary blood flow, respectively, and the blood flow through the constricted coronary artery was not affected by complete constriction of the other coronary arteries. Hence, changes of tone of the more peripheral coronary arteries or arterioles and changes in collateral blood flow are unplausible for the main factor of the fluctuations. A fall in peripheral coronary blood pressure always preceded appearance or augmentation of the ischemic changes in electrocardiogram and left ventricular wall motion, and these ischemic changes returned to the control states after the pressure rose. In addition, the fluctuations could occur or persist without obvious ischemic change on the electrocardiogram and wall motion on several occasions. These findings indicate that the cyclic fluctuations were not secondary to changes in degree of myocardial ischemia.

Intravenous injections of phentolamine, propranolol and atropine could not eliminate the fluctuations. Also, cervical vagotomy and stellectomy could not eliminate the fluctuations. Furthermore, the fluctuations were produced even after surgical denervation. The fact suggests that neither cardiac sympathetic nerves nor vagus nerves were responsible for the cyclic fluctuations.

Nitroglycerin can suppress contraction of the isolated coronary smooth muscles which is caused by potassium ions or acetylcholine. ${ }^{1)}$ This agent suppressed the cyclic fluctuations in coronary blood pressure and flow in this study. Suppression of the fluctuations occurred when the agent was injected during the phase of rise, but not during the phase of fall. A dose sufficient to affect the coronary smooth muscles may have entered into the partially constricted coronary artery when the injection was performed during the phase of rise since the flow was restored considerably in this phase. All these findings indicate that the cyclic fluctuations in blood pressure and flow of the partially constricted coronary artery were due to cyclic changes in coronary smooth muscle tone which were independent of nervous control. However, it remained to be elucidated whether other unknown mechanisms have been involved in initiation and maintenance of the cyclic fluctuations.

The period duration of the cyclic fluctuations observed in this study was of the minute range in the majority of experiments, and was close to that of spontaneous contraction of the taenia coli ${ }^{2}$ and spontaneous fluctuations in 
human skeletal muscle blood flow. ${ }^{3}$

Cyclic appearance of elevation of the ST segment of electrocardiogram associated with fluctuations in coronary blood pressure and flow resembles cyclic attacks of Prinzmetal's variant form of angina pectoris. ${ }^{4), 5)}$ Period duration of fluctuations observed in this study was close to that of cyclic anginal episodes.5) In addition, recent clinical investigations revealed frequent occurrence of transient spasm of the main coronary artery in this type of angina pectoris. ${ }^{6)}$ However, it remains to be elucidated whether an identical mechanism is involved in the clinical and experimental events.

\section{REFERENCES}

1. Norton JM, Gellai M, Detar R: Effect of adenosine on isolated coronary vascular smooth muscle. Pfügers Arch Physiol 335: 279, 1972

2. Wienbeck M, Golenhofen K, Lammel E: Spontanaktivität von isolierter glatter Muskulatur. Pflügers Arch Physiol 300: 70, 1968

3. Golenhofen K, Hildebrandt G: Über spontan rhythmische Schwankungen der Muskeldurchblutung des Menschen. Ztsch Kreislaufforsch 46: 257, 1957

4. Prinzmetal M, Kenner R, Merles ET et al: Angina pectoris. I. A variant form of angina pectoris. Am J Med 27: 375, 1959

5. Murao S, Harumi K, Mashima $\mathrm{S}$ et al: Clinical and experimental study on the mechanism of Prinzmetal's, variant angina. J Jap Soc Intern Med 63: 751, 1974

6. Dhürendhar RV, Watt DL, Silver MD et al: Prinzmetal's, variant form of angina pectoris with arteriographic evidence of coronary artery spasm. Am J Ciardiol 30: 902, 1972 